\title{
ACÓRDÃO N. 33586
}

PRESTAÇÃO DE CONTAS (11531) N. 0601663-30.2018.6.24.0000 FLORIANÓPOLIS

Relator: Juiz Celso Kipper

Requerente: Eleição 2018 - Silvio Dreveck - Deputado Estadual

- ELEIÇÕES 2018 - PRESTAÇÃO DE CONTAS - CANDIDATO A DEPUTADO ESTADUAL.

- OMISSÃO DE DESPESAS ENCONTRADAS NA BASE DE DADOS DA JUSTIÇA ELEITORAL - A) NOTAS FISCAIS SUPOSTAMENTE EMITIDAS EM DUPLICIDADE - COMPROVAÇÃO DE QUE APENAS UMA DELAS FOI CANCELADA - OMISSÃO NÃO AFASTADA QUANTO ÀS DEMAIS - IRREGULARIDADE DE VALOR ÍNFIMO, QUE CORRESPONDE A 0,05\% DO TOTAL DE DESPESAS FINANCEIRAS - ANOTAÇÃO DE RESSALVA - B) IMPULSIONAMENTO DE CONTEÚDOS CONTRATADOS COM O FACEBOOK - DESPESAS LANÇADAS NA PRESTAÇÃO DE CONTAS COM O REGISTRO DE VALORES E DOCUMENTOS DIVERSOS - INEXISTÊNCIA DE OMISSÃO - NOTAS FISCAIS EMITIDAS PELO FACEBOOK, RESPALDANDO A QUASE TOTALIDADE DAS DESPESAS - PEQUENA PARTE DOS DISPÊNDIOS DECLARADOS NÃO AMPARADA EM DOCUMENTOS FISCAIS - IRREGULARIDADE DE PEQUENA MONTA, QUE REPRESENTA MENOS DE 0,01\% DO TOTAL DE DESPESAS FINANCEIRAS DE CAMPANHA - ANOTAÇÃO DE RESSALVA - DEVOLUÇÃO DO VALOR NÃO COMPROVADO MEDIANTE DOCUMENTO FISCAL AO TESOURO NACIONAL - VALOR IRRISÓRIO - DESNECESSIDADE - C) DOCUMENTO FISCAL EMITIDO POR POSTO DE COMBUSTÍVEL CONTRATADO PARA A CAMPANHA - ALEGAÇÃO DE DESCONHECIMENTO DA DESPESA E DE QUE A NOTA FISCAL NÃO TERIA SIDO APRESENTADA PELO FORNECEDOR ATÉ A ENTREGA DA PRESTAÇÃO DE CONTAS - RESPONSABILIDADE PELOS GASTOS DE CAMPANHA ATRI- 
BUÍDAS PELA LEGISLAÇÃO AO CANDIDATO - OMISSÃO NÃO AFASTADA - GASTO QUE REPRESENTA 0,12\% DAS DESPESAS FINANCEIRAS DE CAMPANHA - APLICAÇÃO DOS PRINCÍPIOS DA PROPORCIONALIDADE E DA RAZOABILIDADE - ANOTAÇÃO DE RESSALVAS.

- PAGAMENTO, COM RECURSOS DO FUNDO PARTIDÁRIO, DE DESPESA QUE NÃO SE ENQUADRA NAS HIPÓTESES DE GASTOS COM VERBA DESSA NATUREZA - DECORAÇÃO COM BALÕES - GASTO COM EVENTO DESTINADO À PROMOÇÃO DE CANDIDATURA - POSSIBILIDADE PREVISTA NO ART. 37, IX, DA RESOLUÇÃO TSE N. 23.553/2017 - REGULARIDADE DO DISPÊNDIO RECONHECIDA PELO ÓRGÃO TÉCNICO - IRREGULARIDADE AFASTADA.

- INCONSISTÊNCIAS NAS DESPESAS PAGAS COM RECURSOS DO FUNDO ESPECIAL DE FINANCIAMENTO DE CAMPANHA (FEFC) - A) DESPESA DESCRITA COMO "PALESTRA GERENCIAL SOBRE MOBILIZAÇÃO E USO DE REDES SOCIAIS EM CAMPANHAS” INEXISTÊNCIA, NA LEGISLAÇÃO, DE PREVISÃO DE DESPESAS QUE PODEM OU NÃO SER REALIZADAS COM RECURSOS DO FEFC OU COM RECURSOS PÚBLICOS, SALVO RARAS EXCEÇÕES REFERENTES ÀS VERBAS DO FUNDO PARTIDÁRIO, QUE NÃO GUARDAM RELAÇÃO COM O GASTO QUESTIONADO APLICAÇÃO DAS NORMAS QUE ELENCAM OS GASTOS QUE SE CARACTERIZAM COMO ELEITORAIS - CASO CONCRETO - CONTRATAÇÃO DE PALESTRA COM TEMA PERTINENTE À PROPAGANDA ELEITORAL MEDIANTE IMPULSIONAMENTO DE CONTEÚDOS NAS REDES SOCIAIS - FORMA DE PROPAGANDA DECORRENTE DE INOVAÇÃO LEGISLATIVA - VEDAÇÃO À CONTRATAÇÃO DE INTERMEDIÁRIO PARA A REALIZAÇÃO DO IMPULSIONAMENTO ART. 26, XV, DA LEI N. 9.504/1997 E ART. 37, XII, DA RESOLUÇÃO TSE N. 23.553/2017 - POSSIBILIDADE DE REMUNERAÇÃO OU GRATIFICAÇÃO DE QUALQUER 
ESPÉCIE A PESSOAL QUE PRESTE SERVIÇOS A CANDIDATOS E A PARTIDOS POLÍTICOS - ART. 26, VII, DA LEI N. 9.504/1997 E ART. 37, VII, DA RESOLUÇÃO TSE

N. 23.553/2017 - DESPESA ELEITORAL CARACTERIZA- DA POSSIBILIDADE DE PAGAMENTO COM RECUR-SOS DO FUNDO ESPECIAL DE FINANCIAMENTO DE CAMPANHA IRREGULARIDADE AFASTADA - B) NÃOAPRESENTAÇÃO DE DOCUMENTO FISCAL PARA COMPROVAR A REALIZAÇÃO DE DESPESA COM IM-PULSIONAMENTO DE CONTEÚDOS APRESENTA- ÇÃO DAS NOTAS FISCAIS CORRESPONDENTES AOS GASTOS INICIALMENTE CONTABILIZADOS COM REFERÊNCIA A BOLETOS BANCÁRIOS - IRREGULA-RIDADE SANADA.

- CONTAS APROVADAS COM RESSALVAS. 